\title{
Evaluation of radial distribution of cartilage degeneration and necessity of pre-contrast measurements using radial dGEMRIC in adults with acetabular dysplasia
}

\author{
Li Xu ${ }^{1,2}$, Yongbin Su${ }^{1}$, Karl-Philipp Kienle ${ }^{3}$, Daichi Hayashi ${ }^{2}$, Ali Guermazi ${ }^{2}$, Jing Zhang ${ }^{1}$, Yongming Dai ${ }^{4}$ \\ and Xiaoguang Cheng ${ }^{1 *}$
}

\begin{abstract}
Background: The purpose of the present study was to investigate the radial distribution patterns of cartilage degeneration in dysplastic hips at different stages of secondary osteoarthritis (OA) by using radial delayed gadolinium-enhanced magnetic resonance imaging of cartilage (dGEMRIC), and to assess whether pre-contrast measurements are necessary.
\end{abstract}

Methods: Thirty-five hips in 21 subjects (mean age \pm SD, $27.6 \pm 10.8$ years) with acetabular dysplasia (lateral CE angle $<25^{\circ}$ ) were studied. Severity of OA was assessed on radiographs using Tönnis grading. Pre- (T1 pre) and post-contrast T1 $\left(\mathrm{T} 1_{\mathrm{Gd}}\right)$ values were measured at 7 sub-regions on radial reformatted slices acquired from a 3-dimensional (3D) $\mathrm{T} 1$ mapping sequence using a $1.5 T$ MR scanner. Values of radial $T 1_{\text {pre, }} T 1_{G d}$ and $\Delta R 1\left(1 / T 1_{G d}-1 / T 1_{\text {pre }}\right)$ of subgroups with different severity of OA were compared to those of the subgroup without OA using nonparametric tests, and bivariate linear Pearson correlations between radial $T 1_{\mathrm{Gd}}$ and $\triangle \mathrm{R} 1$ were analyzed for each subgroup.

Results: Compared to the subgroup without $O A$, the subgroup with mild $O A$ was observed with a significant decrease in $\mathrm{T1}_{\mathrm{Gd}}$ in the anterosuperior to superior sub-regions (mean, $476 \sim 507 \mathrm{~ms}, p=0.026 \sim 0.042$ ) and a significant increase in $\Delta \mathrm{R} 1$ in the anterosuperior to superoposterior and posterior sub-regions (mean, $0.93 \sim 1.37 \mathrm{~s}^{-1}, p=0.012 \sim 0.042$ ). The subgroup with moderate to severe OA was observed with a significant overall decrease in $\mathrm{T}_{\mathrm{Gd}}$ (mean, $404 \sim 452 \mathrm{~ms}$, $p=0.001 \sim 0.020$ ) and an increase in $\Delta \mathrm{R} 1$ (mean, $1.17 \sim 1.69 \mathrm{~s}^{-1}, p=0.001 \sim 0.020$ ). High correlations were observed between radial $\mathrm{T}_{\mathrm{Gd}}$ and $\Delta \mathrm{R} 1$ for all subgroups $(r=-0.869 \sim-0.944, p<0.001)$.

Conclusions: Radial dGEMRIC without pre-contrast measurements is useful for evaluating different patterns of cartilage degeneration in the entire hip joint of patients with hip dysplasia, particularly for those in early stages of secondary OA.

Keywords: Acetabular dysplasia, Cartilage degeneration, Radial magnetic resonance imaging, dGEMRIC

\section{Background}

Hip osteoarthritis $(\mathrm{OA})$ is one of the major causes of musculoskeletal disability among adults [1]. One of the common anatomical predisposing factors for hip OA, acetabular dysplasia (AD), is seen not only in young patients but also in elderly patients [2]. A shallow acetabulum results in statically elevated contact pressure, reduced

\footnotetext{
*Correspondence: xiao65@263.net

'Department of Radiology, 4th Medical College of Peking University (Beijing Jishuitan Hospital), 31 Xinjiekou East Street, Beijing 100035, China Full list of author information is available at the end of the article
}

contact area, and joint instability, and OA tends to develop much earlier in the population with $A D$ than in those with normal acetabular architecture [3,4]. Hip preserving procedures are desirable solutions especially for young or active patients with AD [5]. They have been widely performed and are accounted as extremely effective treatments for patients without significant secondary OA $[6,7]$. However, the reliability of these procedures in patients with secondary OA remains undefined $[5,6,8]$. The level of articular cartilage degeneration is the most important factor affecting the postoperative results [9].

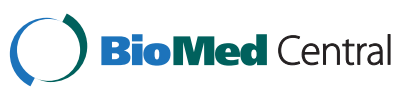


Due to the unreliability of preoperative radiographic measurements, preoperative magnetic resonance imaging (MRI) is needed to assess the cartilage status in these patients [10].

Delayed gadolinium-enhanced magnetic resonance imaging of cartilage (dGEMRIC) is a minimally invasive technique to assess the biochemical properties of articular cartilage. The intravenously injected anionic contrast agent gadopentetate $\left(\right.$ Gd-DTPA $\left.{ }^{2-}\right)$ distributes in cartilage inversely to the concentration of negatively charged glycosaminoglycans (GAGs). GAGs provide cartilage with its compressive stiffness and are lost early during development of OA [11]. The dGEMRIC technique has been shown to be useful for assessing cartilage integrity in dysplastic hips by using coronal T1 mapping sequences $[10,12]$. The radial dGEMRIC is obtained by radial reformation from a 3D data set using dual flip angle T1 mapping sequence. Compared to conventional coronal T1 mapping, the radial dGEMRIC provides radial reformatted slices rotating from anterior to posterior perpendicular to the acetabular rim, allowing evaluation of the cartilage status in various radial regions of the entire hip joint. Distribution of the T1 dGEMRIC values measured using radial dGEMRIC have been found to be unique in different sub-groups of femoroacetabular impingement (FAI) [13]. These patterns of cartilage degeneration, reflected by the radial dGEMRIC index, have not yet been fully investigated on dysplastic hips at different stages of secondary OA.

Currently, post-contrast $\mathrm{T} 1$ relaxation time $\left(\mathrm{T} 1_{\mathrm{Gd}}\right)$ is commonly used as the dGEMRIC index to determine the relative GAG levels within articular cartilage [10,12-14]. An inherent assumption behind this is that pre-contrast $\mathrm{T} 1$ relaxation time $\left(\mathrm{T} 1_{\text {pre }}\right)$ does not vary significantly with the health status of cartilage. However, $\mathrm{T} 1_{\text {pre }}$ values can vary greatly in reparative cartilage and in the native cartilage with fibrillation or edema, compared to normal hyaline cartilage $[15,16]$. The difference between the relaxation rates $\left(\Delta \mathrm{R} 1=1 / \mathrm{T} 1_{\mathrm{Gd}}-1 / \mathrm{T} 1_{\text {pre }}\right)$ showed a better correlation with biopsy-determined GAG content in transplanted cartilage than either $\mathrm{T} 1_{\text {pre }}$ or $\mathrm{T}_{\mathrm{Gd}}$ [16]. In native cartilage, a high correlation between $\mathrm{T} 1_{\mathrm{Gd}}$ and $\Delta \mathrm{R} 1$ was observed in the weight-bearing region of hip and knee joints in asymptomatic volunteers and OA patients [17-19]. The relationship between $\mathrm{T} 1_{\mathrm{Gd}}$ and $\Delta \mathrm{R} 1$ on radial $\mathrm{T} 1$ mapping has not been reported.

Based on the above-mentioned findings, we hypothesized that: radial dGEMRIC would depict different patterns of articular cartilage degeneration in dysplastic hips at different stages of secondary OA; and that pre-contrast imaging is unnecessary for radial dGEMRIC in hips at any stage of secondary OA. The aims of this current study were to investigate, first, the radial distribution of cartilage degeneration using radial dGEMRIC indices, and second, the correlations between radial $\mathrm{T} 1_{\mathrm{Gd}}$ and $\Delta \mathrm{R} 1$ in dysplastic hips at different stages of secondary OA.

\section{Methods \\ Subjects}

Subjects who were referred to our institution for a periacetabular osteotomy because of radiographically diagnosed $\mathrm{AD}$ (lateral center-edge angle of Wiberg, LCE angle $<25^{\circ}$ ) were recruited for this study from March to December 2010. Subjects with other hip diseases or previous hip surgery were excluded. However, subjects with closed reduction during infancy were not excluded. A total of 35 hips (16 left, 19 right) in 21 subjects (19 women, 2 men) ranging from 14 to 54 years (mean age \pm SD, $27.6 \pm 10.8$ years) were included in this prospective study. All subjects were evaluated for clinical symptoms, and underwent both radiographic and MRI assessments. The study protocol was approved by the local ethics committee of Beijing Jishuitan Hospital and each participant signed a written informed consent before examinations.

\section{Clinical and radiographic assessments}

Each hip was individually assessed for pain on 5 items with the Western Ontario and McMaster Universities Osteoarthritis (WOMAC) questionnaire [20], during the interval between pre- and post-contrast imaging sessions. Each item was scored on the Likert scale (0 for no pain to 4 for extreme pain). Pain score was calculated as the summed score of the 5 items for each hip.

A standing antero-posterior pelvic radiograph was performed with the beam centered on the pubic symphysis. The LCE angle, representing the severity of dysplasia, was measured as the angle formed by a vertical line through the center of the femoral head and a line connecting the center of the femoral head and the laterosuperior edge of the acetabulum [21]. The minimum joint space width was measured as the minimum radial distance between the acetabulum and the femoral head in the weight-bearing zone [22]. The severity of secondary OA was determined using Tönnis grading [23]: 0, no arthritis; 1 , bony sclerosis; 2 , small cysts, moderate joint space narrowing; 3, large cysts, severe joint space loss, possible collapse of femoral head. Radiographic measurements were performed by an experienced musculoskeletal radiologist (YBS) using JiveX [dv] DICOM Viewer version 4.3 (VISUS Technology Transfer GmbH, Deutschland), who was blinded to the clinical information of subjects.

\section{MRI protocols and measurements}

MRI was performed in the supine position using a $1.5 \mathrm{~T}$ MRI system (Espree, Siemens, Erlangen, Germany) with a body-matrix phased-array coil. For the contrast-enhanced scan, a double dose $(0.4 \mathrm{mmol} / \mathrm{kg})$ of the gadolinium-based 
contrast agent Magnevist (Gd-DTPA ${ }^{2-}$, Schering, Germany) was administered intravenously. After injection all patients were asked to walk for 15 minutes and to rest for $30 \mathrm{~min}$ utes. The post-contrast scan was thus taken 45 minutes after injection, which is within the recommended time window for dGEMRIC [24]. The MRI protocol included: (1) axial T1-weighted (T1w) turbo spin echo (TSE) (repetition time $(\mathrm{TR})=491 \mathrm{~ms}$, echo time $(\mathrm{TE})=13 \mathrm{~ms}$, slice thickness/ slice gap $3.0 \mathrm{~mm} / 0.3 \mathrm{~mm}$, field of view (FOV) $160 \mathrm{~mm}$, matrix size $512 \times 256$, number of signal averages 1 , acquisition time (TA) $4 \mathrm{~min}$ and $14 \mathrm{sec}$ ); (2) oblique coronal and sagittal proton density-weighted (PDw) TSE (TR = 3060 $\mathrm{ms}, \mathrm{TE}=9.1 \mathrm{~ms}$, slice thickness $/$ slice gap $2.0 \mathrm{~mm} / 0.2 \mathrm{~mm}$, FOV $130 \mathrm{~mm}$, matrix size 256 x 205, number of signal averages 1, TA $5 \mathrm{~min}$ and $35 \mathrm{sec}$ ); (3) 3D isotropic dual-flip angle gradient echo (GRE) sequence utilizing inline T1 measurements both pre- and post-contrast $(\mathrm{TR}=25 \mathrm{~ms}$, $\mathrm{TE}=3.6 \mathrm{~ms}$, flip angles of $10^{\circ}$ and $35^{\circ}$, slice thickness 0.78 $\mathrm{mm}$, FOV $200 \mathrm{~mm}$, matrix size $256 \times 256$, slab $=96$, voxel size $0.78 \mathrm{~mm}^{3}$, TA $8 \mathrm{~min}$ and $46 \mathrm{sec}$ ). The total time for the MRI examination including patients walking and resting after contrast agent injection was between 80 and $85 \mathrm{~min}$.

The 3D T1 dataset was reconstructed using a Leonardo workstation (Siemens, Erlangen, Germany). We reconstructed thirteen radial reformats rotating around the femoral head-neck axis and perpendicular to the acetabular rim, with a slice thickness of $2 \mathrm{~mm}$ at $13.8^{\circ}$ intervals (Figure 1A-C). Guided by the 13 reconstructed slices, the hip joint was divided into 7 radial sub-regions: anterior, anterosuperior, superoanterior, superior, superoposterior, posterosuperior, and posterior. Each radial sub-region included 2 radial slices (Figure $1 \mathrm{D}) . \mathrm{T} 1_{\text {pre }}$ and $\mathrm{T} 1_{\mathrm{Gd}}$ were measured on the 13 radial reformatted slices for each hip, with a region of interest (ROI) involving acetabular and femoral cartilage from the acetabular rim to the acetabular fossa (Figure 1E). Values of the radial $\mathrm{T} 1_{\text {pre }}, \mathrm{T} 1_{\mathrm{Gd}}$ (mean value of the 2 slices in the same radial sub-region) and $\Delta \mathrm{R} 1=1 /$ radial $\mathrm{T} 1_{\mathrm{Gd}}-1 /$ radial $\mathrm{T} 1_{\text {pre }}$ for each radial sub-region were then calculated. Global $\mathrm{T} 1_{\text {pre }}, \mathrm{T} 1_{\mathrm{Gd}}$ and $\Delta \mathrm{R} 1$ of each hip were calculated as the mean values of the radial indices. ROI measurements were performed by a trained radiologist (LX). To assess the intra-observer and inter-observer agreements for radial $\mathrm{T} 1_{\text {pre }}$ and $\mathrm{T} 1_{\mathrm{Gd}}$ measurements, 10 hips were randomly selected one month later and radial $\mathrm{T} 1_{\text {pre }}$ and $\mathrm{T} 1_{\mathrm{Gd}}$ were measured again by the same reader (LX) and another trained radiologist (YBS) on the same reformatted slices as the first measurements. Both readers were blinded to the clinical and radiographic status of the subjects.
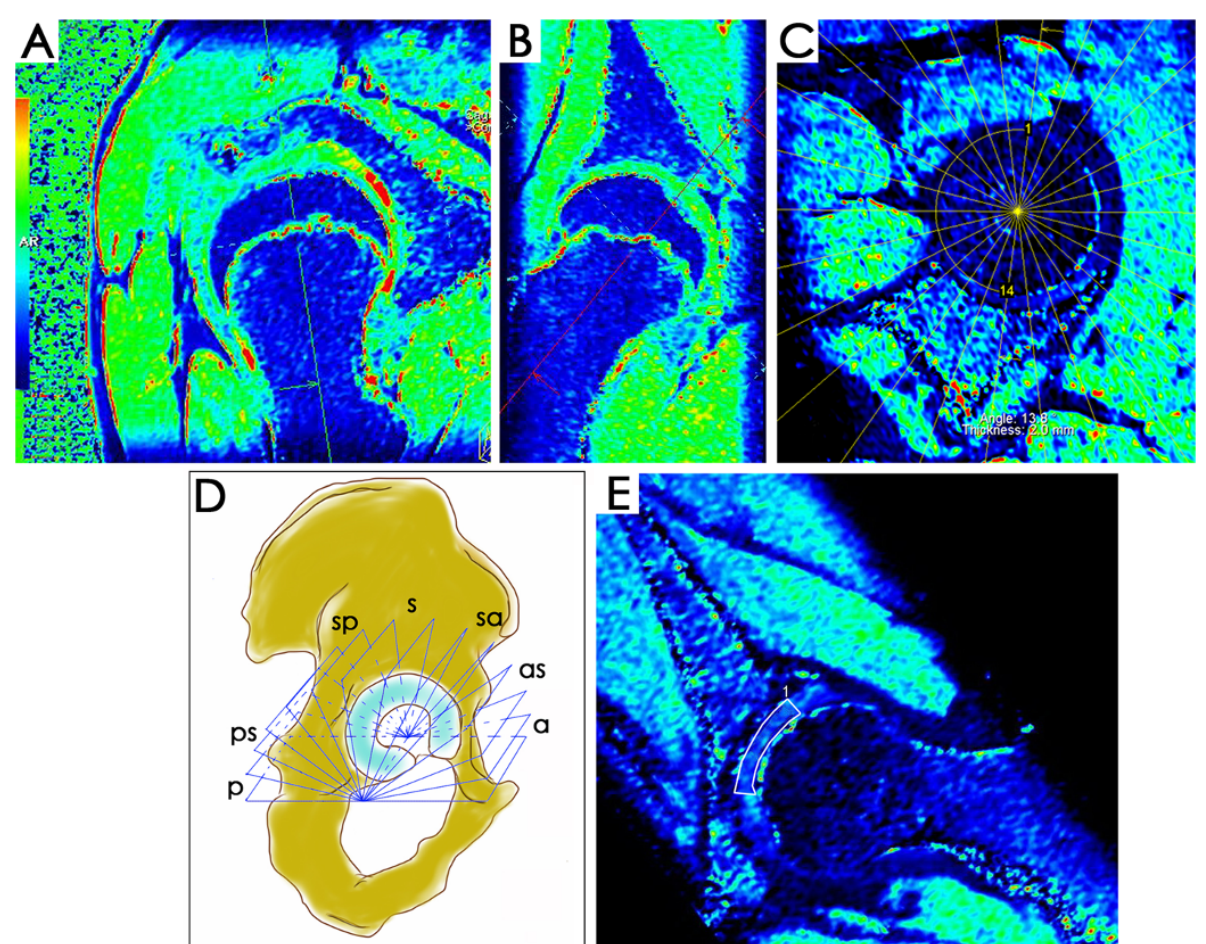

Figure 1 Radial reconstruction of the T1 maps. By alignment of the axis perpendicular to the femoral head-neck from sagittal (A) and coronal (B) views on the 3D viewer, an oblique sagittal view plane (C) was obtained and 13 radial slices with an interval of $13.8^{\circ}$ were generated in the center of the femoral head. For each radial sub-regional $\mathrm{T} 1_{\mathrm{Gd}}$ assessment: 1, anterior; 2, anterosuperior; 3, superoanterior; 4, superior; 5 , superoposterior; 6, posterosuperior and 7, posterior, 2 radial slices were included (D). ROI analysis was performed involving the acetabular and femoral cartilage from acetabular rim to acetabular fossa (E). 


\section{Statistical analyses}

Statistical analyses were performed using SPSS statistical software version 11.5 (SPSS Inc., Chicago, IL, USA). The intra- and inter-observer agreements of radial $\mathrm{T} 1_{\text {pre }}$ and $\mathrm{T} 1_{\mathrm{Gd}}$ measurements were evaluated on 10 random hips (7 ROIs per hip, 70 data points) using intra-class correlation (ICC) analyses.

To investigate the associations of dGEMRIC indices to the severity of lateral $\mathrm{AD}$, the value of global $\mathrm{T} 1_{\mathrm{Gd}}$, global $\Delta \mathrm{R} 1$, radial $\mathrm{T} 1_{\mathrm{Gd}}$ and radial $\Delta \mathrm{R} 1$ was individually calculated using the Pearson correlation coefficient with LCE angle. According to the severity of secondary OA as assessed by the Tönnis grading, all hips were divided into 3 subgroups: no $\mathrm{OA}=$ Tönnis grade 0 ; mild $\mathrm{OA}=$ Tönnis grade 1 ; moderate to severe $\mathrm{OA}=$ Tönnis grade 2 3. Mean values and 95\% confidence intervals (95\% CI) of radial $\mathrm{T} 1_{\text {pre }}, \mathrm{T} 1_{\mathrm{Gd}}$ and $\Delta \mathrm{R} 1$ were calculated for each subgroup and 2-independent samples nonparametric tests (Mann-Whitney U test) were used to compare radial $\mathrm{T} 1_{\text {pre, }} \mathrm{T} 1_{\mathrm{Gd}}$ and $\Delta \mathrm{R} 1$ of subgroup 2 (Tönnis grade 1 ) and subgroup 3 (Tönnis grade $2 \sim 3$ ) to those of subgroup 1 (Tönnis grade 0 ), respectively. To assess the necessity of pre-contrast T1 measurement of hips at different stages of secondary OA, bivariate linear Pearson correlation analysis (two-tailed) between radial $\mathrm{T} 1_{\mathrm{Gd}}$ and $\Delta \mathrm{R} 1$ was individually performed for each subgroup. The significance level in this study was set at $p<0.05$.

\section{Results}

Results of the clinical and radiographic evaluation are summarized in Table 1.

\section{Intra- and inter-observer reliability analyses}

High intra-observer correlation was detected for both radial $\mathrm{T} 1_{\text {pre }}$ (ICC range, $0.894 \sim 0.959$ ) and radial $\mathrm{T} 1_{\mathrm{Gd}}$ (ICC range, $0.813 \sim 0.928$ ) measurements, with mean absolute differences of $52 \mathrm{~ms}(95 \% \mathrm{CI}, 38 \sim 66 \mathrm{~ms} ; p=$ $0.765)$ for the radial $\mathrm{T} 1_{\text {pre }}$ measurement and $37 \mathrm{~ms}(95 \%$ $\mathrm{CI}, 26 \sim 47 \mathrm{~ms} ; p=0.319)$ for the radial $\mathrm{T} 1_{\mathrm{Gd}}$ measurement. Inter-observer correlation was also high for the radial $\mathrm{T} 1_{\text {pre }}$ (ICC range, $0.775 \sim 0.913$ ) and radial $\mathrm{T} 1_{\mathrm{Gd}}$ (ICC range, $0.787 \sim 0.918$ ) measurements. Mean absolute difference between the 2 readers was $64 \mathrm{~ms}(95 \%$ $\mathrm{CI}, 44 \sim 83 \mathrm{~ms} ; p=0.358)$ for radial $\mathrm{T} 1_{\text {pre }}$ measurements and $36 \mathrm{~ms}$ (95\% CI, $27 \sim 44 \mathrm{~ms} ; p=0.299)$ for radial $\mathrm{T} 1_{\mathrm{Gd}}$ measurements.

\section{Radial distribution of dGEMRIC indices}

Mean values and 95\% CI of the radial and global dGEMRIC indices of the study group are shown in Table 2. Moderate correlation was detected between the LCE angle and the global $\mathrm{T} 1_{\mathrm{Gd}}(\mathrm{r}=0.577, p<0.001)$ as well as radial $\mathrm{T} 1_{\mathrm{Gd}}$ in the weight-bearing sub-regions (superoanterior, superior, and superoposterior) $(r=0.578 \sim$

\section{Table 1 Clinical and radiographic evaluations of the study cohort}

\begin{tabular}{|c|c|c|}
\hline Variables & & Study cohort $(n=35)$ \\
\hline \multicolumn{3}{|l|}{ Pain Score } \\
\hline \multirow[t]{3}{*}{ (mean $\pm S D, 4 \pm 3$; range, 0 14) } & $0 \sim 5$ & $28(80 \%)$ \\
\hline & $6 \sim 9$ & $5(14 \%)$ \\
\hline & $\geq 10$ & $2(6 \%)$ \\
\hline \multicolumn{3}{|l|}{ LCE Angle $\left(^{\circ}\right)$} \\
\hline \multirow[t]{3}{*}{ (mean $\pm S D, 8 \pm 12 ;$ range, $-17 \sim 23$ ) } & $<5$ & $11(31 \%)$ \\
\hline & $5 \sim 19$ & $18(51 \%)$ \\
\hline & $20 \sim 25$ & $6(17 \%)$ \\
\hline \multicolumn{3}{|l|}{ Joint Space Width (mm) } \\
\hline \multirow[t]{3}{*}{$($ mean $\pm S D, 5.2 \pm 1.5 ;$ range, $2.8 \sim 10.1)$} & $<4$ & $6(17 \%)$ \\
\hline & $4 \sim 6$ & $21(60 \%)$ \\
\hline & $>6$ & $8(23 \%)$ \\
\hline \multicolumn{3}{|l|}{ Tönnis Grade } \\
\hline & 0 & $6(17 \%)$ \\
\hline & 1 & $21(60 \%)$ \\
\hline & 2 & $7(20 \%)$ \\
\hline & 3 & $1(3 \%)$ \\
\hline
\end{tabular}

0.619, $p<0.001$ ) (Table 2). A similar correlation was detected between the LCE angle and the global $\Delta \mathrm{R} 1$ ( $\mathrm{r}=$ $-0.553, p<0.001)$ as well as radial $\Delta \mathrm{R} 1$ in the weightbearing sub-regions (superoanterior, superior and superoposterior) $(\mathrm{r}=-0.542 \sim-0.632, p<0.001)$ (Table 2$)$.

Subgroup analyses showed different patterns of dGEMRIC indices distribution in various radial subregions. $\mathrm{T} 1_{\text {pre }}$ ranged from 856 to $980 \mathrm{~ms}$ (95\% CI, 800 $\sim 1140 \mathrm{~ms}$ ) in hips without OA. Compared to that subgroup, a significant increase in $\mathrm{T} 1_{\text {pre }}$ was detected in the superoanterior $(p=0.036)$ and posterior $(p=0.022)$ subregions in hips with mild $\mathrm{OA}$ and only in the posterior sub-region $(p=0.001)$ in hips with moderate to severe OA (Figure 2A).

Using the lower limit of the normal range of $\mathrm{T} 1_{\mathrm{Gd}}$ (480 ms) in articular cartilage of the hip joint as the reference standard [12], radial $\mathrm{T} 1_{\mathrm{Gd}}$ were found at normal levels in all sub-regions except the posterior in hips without OA, but decreased into the abnormal range in the anterior to superior sub-regions as well as in the posterior in hips with mild OA (Figure 2B). Compared to the hips without $\mathrm{OA}$, a significant decrease in radial $\mathrm{T} 1_{\mathrm{Gd}}$ was observed in the anterosuperior $(p=0.042)$, superoanterior $(p=0.026)$ and superior $(p=0.031)$ subregions in hips with mild OA. In hips with moderate to severe OA, a significant overall decrease $(p=0.001 \sim$ 0.020 ) in radial $\mathrm{T} 1_{\mathrm{Gd}}$ was observed compared to the hips without OA, with an upper limit of $95 \%$ CI lower than $480 \mathrm{~ms}$ in most sub-regions (anterior, anterosuperior, superoanterior, superior, posterior) (Figure 2B). 
Table 2 Measurements of the dGEMRIC indices and the correlation coefficients between dGEMRIC indices and LCE angle

\begin{tabular}{|c|c|c|c|c|c|c|c|c|c|}
\hline \multirow{3}{*}{\multicolumn{2}{|c|}{ dGEMRIC indices }} & \multicolumn{8}{|c|}{$\mathrm{n}=35$} \\
\hline & & \multicolumn{7}{|c|}{ Radial sub-region } & \multirow[t]{2}{*}{ Global } \\
\hline & & $a$ & as & sa & $s$ & sp & ps & $p$ & \\
\hline \multirow[t]{4}{*}{$T 1_{\text {pre }}$} & Mean (ms) & 927 & 919 & 925 & 948 & 968 & 1021 & 1072 & 969 \\
\hline & $95 \% \mathrm{Cl}$ (ms) & 892 963 & 884 953 & 890 960 & 910 987 & $932 \sim 1004$ & $973 \sim 1069$ & $1007 \sim 1137$ & $941 \sim 996$ \\
\hline & Minimum (ms) & 708 & 786 & 714 & 777 & 832 & 787 & 817 & 838 \\
\hline & Maximum (ms) & 1313 & 1177 & 1179 & 1213 & 1370 & 1477 & 1783 & 1165 \\
\hline \multirow[t]{5}{*}{$\mathrm{T} 1_{\mathrm{Gd}}$} & Mean (ms) & 501 & 490 & 479 & 504 & 526 & 515 & 445 & 494 \\
\hline & $95 \% \mathrm{Cl}$ (ms) & $464 \sim 539$ & $452 \sim 527$ & $443 \sim 515$ & $461 \sim 546$ & 493 558 & $482 \sim 548$ & $416 \sim 474$ & $464 \sim 525$ \\
\hline & Minimum (ms) & 272 & 282 & 318 & 278 & 369 & 265 & 291 & 336 \\
\hline & Maximum (ms) & 736 & 667 & 689 & 740 & 737 & 718 & 598 & 681 \\
\hline & $\begin{array}{l}\text { Correlations }{ }^{a} \text { with } \\
L C E, r\end{array}$ & $\begin{array}{c}0.498 \\
(p=0.002)\end{array}$ & $\begin{array}{c}0.525 \\
(p=0.001)\end{array}$ & $\begin{array}{c}0.578 \\
(p<0.001)\end{array}$ & $\begin{array}{c}0.619 \\
(p<0.001)\end{array}$ & $\begin{array}{c}0.598 \\
(p<0.001)\end{array}$ & $\begin{array}{c}0.405 \\
(p=0.016)\end{array}$ & $\begin{array}{c}0.178 \\
(p=0.306)\end{array}$ & $\begin{array}{c}0.577 \\
(p<0.001)\end{array}$ \\
\hline \multirow[t]{5}{*}{$\overline{\Delta \mathrm{R} 1}$} & Mean $\left(s^{-1}\right)$ & 1.00 & 1.05 & 1.10 & 1.04 & 0.92 & 1.02 & 1.37 & 1.07 \\
\hline & $95 \% \mathrm{Cl}\left(\mathrm{s}^{-1}\right)$ & $0.84 \sim 1.16$ & $0.87 \sim 1.24$ & $0.92 \sim 1.27$ & $0.85 \sim 1.23$ & $0.79 \sim 1.05$ & $0.86 \sim 1.19$ & $1.19 \sim 1.55$ & $0.93 \sim 1.21$ \\
\hline & $\operatorname{Minimum}\left(\mathrm{s}^{-1}\right)$ & 0.33 & 0.40 & 0.21 & 0.10 & 0.31 & 0.29 & 0.55 & 0.45 \\
\hline & Maximum $\left(s^{-1}\right)$ & 2.56 & 2.69 & 2.08 & 2.52 & 1.61 & 3.05 & 2.62 & 1.99 \\
\hline & $\begin{array}{l}\text { Correlations }{ }^{a} \text { with } \\
L C E, r\end{array}$ & $\begin{array}{c}-0.392 \\
(p=0.020)\end{array}$ & $\begin{array}{c}-0.439 \\
(p=0.008)\end{array}$ & $\begin{array}{c}-0.542 \\
(p=0.001)\end{array}$ & $\begin{array}{c}-0.586 \\
(p<0.001)\end{array}$ & $\begin{array}{c}-0.632 \\
(p<0.001)\end{array}$ & $\begin{array}{c}-0.446 \\
(p=0.007)\end{array}$ & $\begin{array}{c}-0.219 \\
(p=0.205)\end{array}$ & $\begin{array}{c}-0.553 \\
(p=0.001)\end{array}$ \\
\hline
\end{tabular}

${ }^{a}$ Correlation was calculated as Pearson correlation coefficient.

The mean value of radial $\Delta \mathrm{R} 1$ ranged from 0.49 to $0.95 \mathrm{~s}^{-1}\left(95 \% \mathrm{CI}, 0.25 \sim 1.27 \mathrm{~s}^{-1}\right)$ in hips without OA, 0.92 to $1.37\left(95 \% \mathrm{CI}, 0.74 \sim 1.61 \mathrm{~s}^{-1}\right)$ in hips with mild $\mathrm{OA}$, and 1.17 to $1.69 \mathrm{~s}^{-1}$ (95\% CI, $0.86 \sim 2.08 \mathrm{~s}^{-1}$ ) in hips with moderate to severe OA (Figure 2C). Compared to the hips without OA, a significant increase in $\Delta \mathrm{R} 1$ was observed in the anterosuperior to superoposterior and posterior $(p=0.012 \sim 0.042)$ sub-regions in hips with mild OA and in all sub-regions $(p=0.001 \sim$ 0.020 ) in hips with moderate to severe OA (Figure $2 \mathrm{C}$ ).

\section{Correlation analyses}

A high linear correlation was detected between radial $\mathrm{T} 1_{\mathrm{Gd}}$ and $\Delta \mathrm{R} 1$ in all 3 subgroups. The Pearson correlation coefficient between radial $\mathrm{T} 1_{\mathrm{Gd}}$ and $\Delta \mathrm{R} 1$ was $-0.919(p<0.001)$ in hips without OA (Figure 3A),
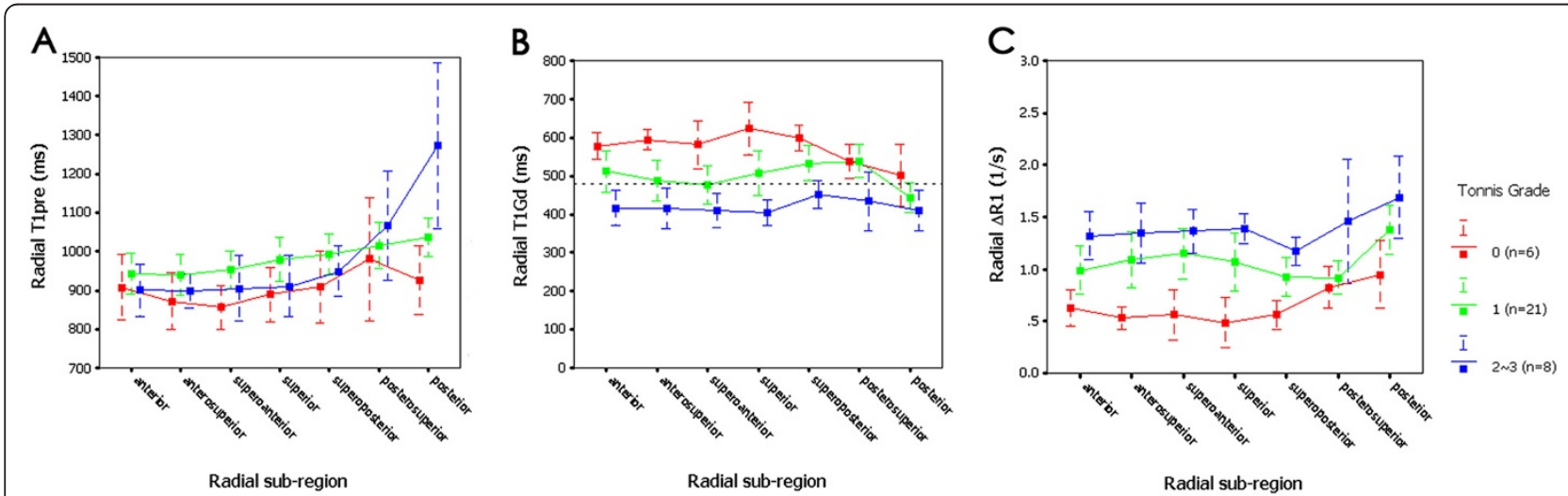

Figure 2 Radial distribution of $\mathrm{T} 1_{\mathrm{pre}}(\mathbf{A}), \mathrm{T} 1_{\mathrm{Gd}}(\mathbf{B})$ and $\Delta \mathrm{R} 1$ (C) according to Tönnis grading. Compared to the Tönnis grade 0 subgroup $T 1_{\text {pre }}$ increased significantly in the superoanterior $(p=0.036)$ and posterior $(p=0.022)$ sub-regions in the Tönnis grade 1 subgroup and in the posterior sub-region $(p=0.001)$ in the Tönnis grade $2 \sim 3$ subgroup; there was a significant decrease in $\mathrm{T} 1_{\mathrm{Gd}}$ in the anterosuperior to superior sub-regions $(p=0.026 \sim 0.042)$ in the Tönnis grade 1 subgroup and a significant overall decrease $(p=0.001 \sim 0.020)$ in the Tönnis grade $2 \sim 3$ subgroup; $\Delta R 1$ increased significantly in the anterosuperior to superoposterior $(p=0.012 \sim 0.042)$ and posterior $(p=0.042)$ sub-regions in the Tönnis grade 1 subgroup and there was a significant overall increase $(p=0.001 \sim 0.020)$ in the Tönnis grade $2 \sim 3$ subgroup. 

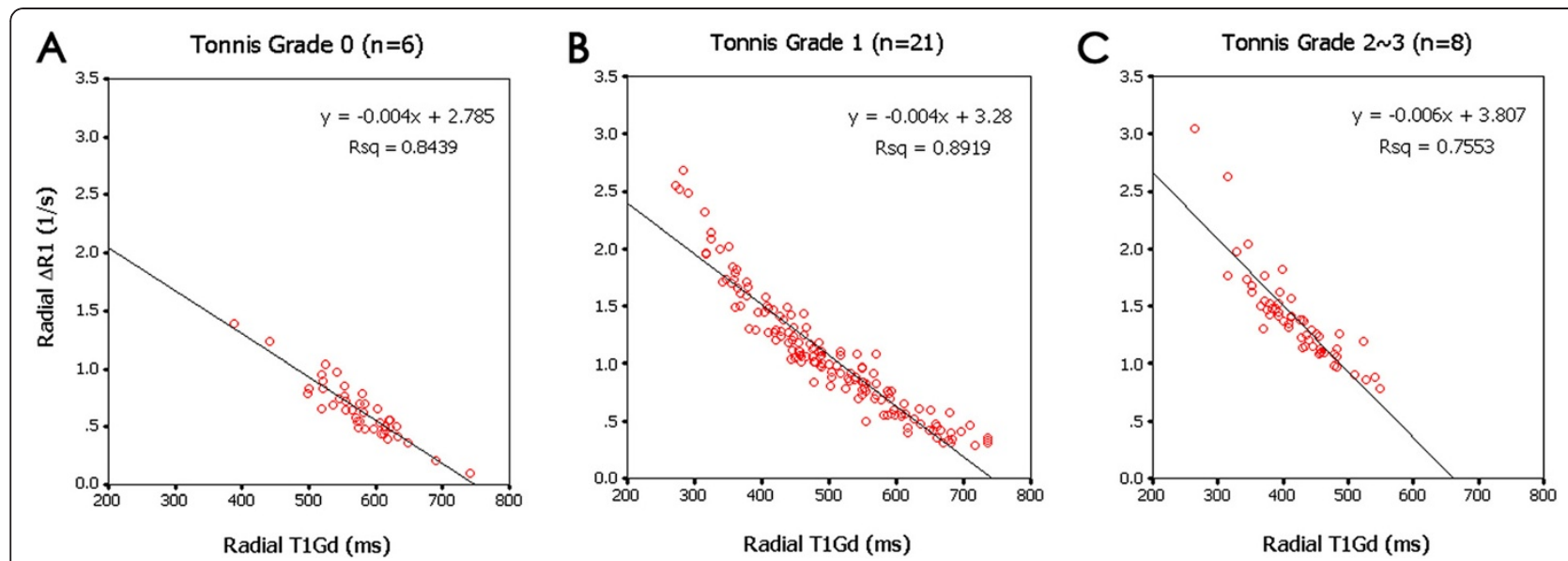

Figure 3 Scatterplot of $\Delta \mathbf{R} 1$ and corresponding radial $\mathbf{T} 1_{\mathrm{Gd}}$. For all 3 subgroups, Tönnis grade $0(\mathbf{A})$, Tönnis grade 1 (B) and Tönnis grade 2 3 (C), high correlations $(r=-0.869 \sim-0.944, p<0.001)$ indicated that $T 1_{\mathrm{Gd}}$ and $\Delta R 1$ are equally effective in evaluating cartilage status. The lines represent the linear regression fit.

$-0.944(p<0.001)$ in hips with mild OA (Figure 3 B), and $-0.869(p<0.001)$ in hips with moderate to severe OA (Figure $3 \mathrm{C}$ ).

\section{Discussion}

In this study, different patterns of $\mathrm{T} 1_{\mathrm{Gd}}$ were shown in various radial sub-regions of dyplastic hip joints in pace with the aggravation of secondary OA. In studies performed on healthy asymptomatic volunteers, $\mathrm{T} 1_{\mathrm{Gd}}$ values were reported as $570 \pm 90 \mathrm{~ms}$ on coronal $\mathrm{T} 1$ mapping by Kim et al. [12], and the mean value of $\mathrm{T} 1_{\mathrm{Gd}}$ ranged from 553.9 to $629.4 \mathrm{~ms}$ on radial $\mathrm{T} 1 \mathrm{mapping}$ according to Bittersohl et al. [25]. Using the values of healthy asymptomatic volunteers as the normal standard [12,25], hips without radiographic $\mathrm{OA}$ in the present study showed a normal mean value of $\mathrm{T}_{\mathrm{Gd}}(538 \sim 624 \mathrm{~ms})$ in the radial sub-regions from anterior to posterosuperior and a mildly decreased $\mathrm{T} 1_{\mathrm{Gd}}$ value (mean, $502 \mathrm{~ms}$ ) in the posterior, with a higher $\mathrm{T} 1_{\mathrm{Gd}}$ value toward the superior sub-regions. In hips with mild radiographic OA a significant decrease in $\mathrm{T} 1_{\mathrm{Gd}}$ was detected in the radial sub-regions from anterosuperior to superior, compared to the hips without radiographic OA. In the hips with moderate to severe radiographic OA, a significant overall decrease in $\mathrm{T}_{\mathrm{Gd}}$ was found compared to those without radiographic OA. The patterns of cartilage degeneration in this series accorded well with the general understanding of cartilage damage in the hip with dysplasia. Previous arthroscopic and radiographic studies have indicated that there is a high prevalence of cartilage lesions in the anterosuperior and superoanterior regions [26,27], and early degenerative processes in dysplastic hips originate at the watershed zone between the acetabular labrum and the acetabular cartilage [28]. Recently, another study using radial dGEMRIC to evaluate cartilage degeneration in hip dysplasia was published by Domayer et al. [14]. Radial dGEMRIC showed an increased T1 in the weightbearing areas of the acetabulum when the dGEMRIC index was more than $500 \mathrm{~ms}$ and a globally decreased $\mathrm{T} 1$ when the dGEMRIC index was less than $500 \mathrm{~ms}$. Those findings corresponded to ours in the subgroups without radiographic $\mathrm{OA}$ and with moderate to severe radiographic OA.

The decrease of $\mathrm{T} 1_{\mathrm{Gd}}$ value in the posterior sub-region in hips without OA and with mild OA detected in the current study is not an unexpected finding. Knowledge of normal variations of GAG content within different regions of the hip joint is critical for defining the "abnormal" $\mathrm{T} 1_{\mathrm{Gd}}$ and should be taken into consideration. GAG content was revealed to be higher in the weight-bearing portions of the hip joint with a gradual decrease toward the inferior regions $[29,30]$. Correspondingly, radial $\mathrm{T} 1_{\mathrm{Gd}}$ shows a zonal variation in adult asymptomatic hip joints, with higher values toward the superior regions and the lowest value noted in the posterior [25]. Further efforts are needed to establish the ideal zonal cutoff value of $\mathrm{T} 1_{\mathrm{Gd}}$ for distinguishing abnormal cartilage from normal.

Whether $\mathrm{T} 1_{\mathrm{Gd}}$ gives information comparable to $\Delta \mathrm{R} 1$ is one of the central issues in dGEMRIC. Watanabe et al. showed that $\triangle \mathrm{R} 1$ had a better correlation with biopsydetermined GAG content in transplanted cartilage than either $\mathrm{T} 1_{\mathrm{pre}}$ or $\mathrm{T} 1_{\mathrm{Gd}}$ alone [16]. In native articular cartilage, $\Delta \mathrm{R} 1$ was equally effective as $\mathrm{T} 1_{\mathrm{Gd}}$ for both $\mathrm{OA}$ and healthy subjects [17-19]. According to the study performed on FAI patients by Bittersohl et al., the correlation between $\Delta \mathrm{R} 1$ and $\mathrm{T} 1_{\mathrm{Gd}}$ within the weight-bearing region of the hip joint was -0.95 in a study cohort with Tönnis grade $0,-0.89$ in Tönnis grade 1 and -0.88 in asymptomatic volunteers [19]. Li et al. compared the ability of $\Delta \mathrm{R} 1$ and $\mathrm{T} 1_{\mathrm{Gd}}$ to differentiate patients with 
knee OA from healthy subjects [17]. In their study, $\Delta \mathrm{R} 1$ and $\mathrm{T} 1_{\mathrm{Gd}}$ were found to be highly correlated $(\mathrm{r}=-0.96)$ and almost identical in terms of effect sizes and areas under receiver operating characteristic curves. Williams et al. also reported a high correlation $(\mathrm{r}=-0.87 \sim-0.96)$ between $\Delta \mathrm{R} 1$ and $\mathrm{T} 1_{\mathrm{Gd}}$ in knee joints with and without symptoms [18]. Another study found that $\mathrm{T} 1_{\text {pre }}$ values were only minimally different in early cartilage degeneration [31]. Our study showed good consistence with those previous studies. No significant difference of $\mathrm{T} 1_{\text {pre }}$ was detected in any radial sub-regions except the superoanterior and posterior in hips with radiographic OA, compared to those without. Radial $\Delta \mathrm{R} 1$ was observed to have similar patterns of radial distribution and a high inverse linear correlation with radial $\mathrm{T} 1_{\mathrm{Gd}}(\mathrm{r}=-0.869 \sim$ $-0.944)$ in all 3 subgroups. Correlation coefficients between $\Delta \mathrm{R} 1$ and $\mathrm{T} 1_{\mathrm{Gd}}$ noted by the present study were in accordance with those $(\mathrm{r}=-0.87 \sim-0.96)$ noted by previous studies [17-19]. Considering the logistical costs in terms of time and effort to acquire $\mathrm{T} 1_{\text {pre }}$ measurements, the results of the present study support that the current practice of measuring $\mathrm{T} 1_{\mathrm{Gd}}$ is adequate for assessing native cartilage in AD patients using radial dGEMRIC.

The radial dGEMRIC index, with the potential to reflect cartilage status in the entire hip joint, should be effective and helpful for preoperative evaluation in adult $\mathrm{AD}$ patients. The different patterns of $\mathrm{T} 1_{\mathrm{Gd}}$ in various radial sub-regions shown in this current study indicate that the articular cartilage from anterior to superior and posterior is vulnerable to degenerative processes, and therefore evaluation of cartilage status in these subregions is critical for predicting the postoperative effects in cases with early stages of secondary OA. In contrast, significant overall cartilage damage with advanced OA presages a poor postoperative outcome.

The main limitation of the present study was the lack of a healthy control group, for ethical reasons, and therefore the normal zonal variations of dGEMRIC indices are not available. It may not be accurate enough to define "abnormal" radial $\mathrm{T} 1_{\mathrm{Gd}}$ using the reported normal range of $\mathrm{T} 1_{\mathrm{Gd}}$ value measured on coronal $\mathrm{T} 1$ mapping as the normal standard, particularly for the inferior subregions. For the same reason, subgroup comparisons were only performed between hips with radiographic $\mathrm{OA}$ and those without instead of healthy asymptomatic hips. It is possible that the decrease in $\mathrm{T} 1_{\mathrm{Gd}}$ in hips with radiographic OA was underestimated. However, values of radial $\mathrm{T} 1_{\mathrm{Gd}}$ in hips without radiographic $\mathrm{OA}$ in this study were found to be comparable with those in healthy volunteers reported by a previous study [25]. So we believe the issue should have a minimal impact on our conclusion on subgroup comparisons. Future studies should involve healthy asymptomatic volunteers to establish the normal range of radial $\mathrm{T} 1_{\mathrm{Gd}}$ and the best cutoff values for distinguishing abnormal cartilage from normal. In addition, the present study included a relatively inhomogeneous cohort with wide age range (14 to 54 years) and therefore, age related primary OA may be the potential cofounding. Statistically, bilateral observations in part of the study samples may be not strict although no significant correlations ( $p=0.122 \sim 0.994)$ of the radial indices were detected between two hips in the same patient. As in previous studies as described by Kim et al. and Bittersohl et al. [12,25], differentiation between acetabular and femoral cartilage was not possible for the ROI analysis on a $1.5 \mathrm{~T}$ scanner because of the limitation of spatial resolution. Joint fluid as well as Gd-DTPA ${ }^{2-}$ in the synovial fluid may have altered the T1 value.

\section{Conclusions}

Different patterns of cartilage degeneration were detected in dysplastic hips at different stages of secondary OA by radial dGEMRIC indices, with a significant decline of cartilage function in the radial sub-regions from anterosuperior to superior in those with mild radiographic OA, and overall cartilage damage in those with moderate to severe radiographic OA. Because of the high correlations between $\Delta \mathrm{R} 1$ and $\mathrm{T} 1_{\mathrm{Gd}}$, pre-contrast measurements seem unnecessary for radial dGEMRIC regardless of the severity of OA. With the ability to reflect cartilage status in the entire hip joint, radial dGEMRIC seems to be a more useful technique than conventional coronal imaging for preoperative evaluation in $\mathrm{AD}$ patients at an early stage of secondary OA.

\section{Abbreviations}

OA: Osteoarthritis; AD: Acetabular dysplasia; MRI: Magnetic resonance imaging; dGEMRIC: Delayed gadolinium-enhanced magnetic resonance imaging of cartilage; Gd-DTPA ${ }^{2-}$ : Gadopentetate; GAGs: Glycosaminoglycans; 3D: 3-dimensional; FAl: Femoroacetabular impingement; $\mathrm{T} 1_{\mathrm{Gd}}$ : Post-contrast $\mathrm{T} 1$ relaxation time; $\mathrm{T} 1_{\text {pre: }}$ Pre-contrast $\mathrm{T} 1$ relaxation time; $\Delta \mathrm{R} 1$ : Difference between the relaxation rates; LCE angle: Lateral center-edge angle of Wiberg; WOMAC: Western Ontario and McMaster Universities Osteoarthritis; a: anterior; as: anterosuperior; sa: superoanterior; s: superior; sp: superoposterior; ps: posterosuperior; p: posterior; ROI: region of interest; ICC: Intra-class correlation; Cl: Confidence intervals.

\section{Competing interests}

Ali Guermazi is the President of Boston Imaging Core Lab, LLC, and is a Consultant to Merck Serono, Genzyme, Stryker, AstraZeneca, and Novartis. Yongming Dai is employed by Siemens Healthcare. No other authors have any financial disclosures.

\section{Authors' contributions}

All authors contributed substantially to drafting and revising the intellectual contents of the manuscript and approved the final version for submission: study design, K-PK, JZ and LX; data collection and magnetic resonance images measurement, LX and YS; data analysis, $\mathrm{LX}$ and $\mathrm{DH}$; writing of the initial draft of the manuscript, $L X, D H, A G$, and $X C$; technical supporting, $Y D$; guarantor of the integrity of the study: $X C$. All authors read and approved the final manuscript.

\section{Acknowledgements}

The authors would like to thank the study participants for contributing their time and efforts. 


\section{Role of funding source}

Technical assistance with the imaging protocol was obtained from Siemens Healthcare Co, Ltd. However, they were not involved in the analysis, interpretation of results or writing of this paper.

\section{Author details}

'Department of Radiology, 4th Medical College of Peking University (Beijing Jishuitan Hospital), 31 Xinjiekou East Street, Beijing 100035, China. ${ }^{2}$ Department of Radiology, Boston University School of Medicine, FGH Building 3rd Floor, 820 Harrison Avenue, Boston, MA 02118, USA. ${ }^{3}$ Department of Orthopedic Surgery, University of Bern, Bern, Switzerland. 4Siemens Healthcare China, MR Collaborations NE Asia, Shanghai 201318, China.

Received: 13 March 2012 Accepted: 11 October 2012 Published: 30 October 2012

\section{References}

1. Felson DT, Zhang Y: An update on the epidemiology of knee and hip osteoarthritis with a view to prevention. Arthritis Rheum 1998, 41(8):1343-1355.

2. Reijman M, Hazes JM, Pols HA, Koes BW, Bierma-Zeinstra SM: Acetabular dysplasia predicts incident osteoarthritis of the hip: the Rotterdam study. Arthritis Rheum 2005, 52(3):787-793.

3. Hipp JA, Sugano N, Millis MB, Murphy SB: Planning acetabular redirection osteotomies based on joint contact pressures. Clin Orthop Relat Res 1999, 364:134-143.

4. Russell ME, Shivanna KH, Grosland NM, Pedersen DR: Cartilage contact pressure elevations in dysplastic hips: a chronic overload model. J Orthop Surg Res 2006, 1:6.

5. Trousdale RT, Ekkernkamp A, Ganz R, Wallrichs SL: Periacetabular and intertrochanteric osteotomy for the treatment of osteoarthrosis in dysplastic hips. J Bone Joint Surg Am 1995, 77(1):73-85.

6. Murphy S, Deshmukh R: Periacetabular osteotomy: preoperative radiographic predictors of outcome. Clin Orthop Relat Res 2002, 405:168-174.

7. Takatori Y, Ninomiya S, Nakamura S, Morimoto S, Moro T, Nagai I, Mabuchi A: Long-term results of rotational acetabular osteotomy in patients with slight narrowing of the joint space on preoperative radiographic findings. J Orthop Sci 2001, 6(2):137-140.

8. Clohisy JC, Schutz AL, St John L, Schoenecker PL, Wright RW: Periacetabular osteotomy: a systematic literature review. Clin Orthop Relat Res 2009, 467(8):2041-2052

9. Yasunaga $Y$, Ikuta $Y$, Kanazawa $T$, Takahashi $K$, Hisatome $T$ : The state of the articular cartilage at the time of surgery as an indication for rotational acetabular osteotomy. J Bone Joint Surg Br 2001, 83(7):1001-1004.

10. Cunningham T, Jessel R, Zurakowski D, Millis MB, Kim YJ: Delayed gadolinium-enhanced magnetic resonance imaging of cartilage to predict early failure of Bernese periacetabular osteotomy for hip dysplasia. J Bone Joint Surg Am 2006, 88(7):1540-1548.

11. Sweet MB, Thonar EJ, Immelman AR, Solomon L: Biochemical changes in progressive osteoarthrosis. Ann Rheum Dis 1977, 36(5):387-398.

12. Kim YJ, Jaramillo D, Millis MB, Gray ML, Burstein D: Assessment of early osteoarthritis in hip dysplasia with delayed gadolinium-enhanced magnetic resonance imaging of cartilage. J Bone Joint Surg Am 2003, 85-A(10):1987-1992.

13. Bittersohl B, Steppacher S, Haamberg T, Kim YJ, Werlen S, Beck M, Siebenrock KA, Mamisch TC: Cartilage damage in femoroacetabular impingement (FAl): preliminary results on comparison of standard diagnostic vs delayed gadolinium-enhanced magnetic resonance imaging of cartilage (dGEMRIC). Osteoarthr Cartil 2009, 17(10):1297-1306.

14. Domayer SE, Mamisch TC, Kress I, Chan J, Kim YJ: Radial dGEMRIC in developmental dysplasia of the hip and in femoroacetabular impingement: preliminary results. Osteoarthr Cartil 2010, 18(11):1421-1428.

15. Tiderius CJ, Olsson LE, Leander P, Ekberg O, Dahlberg L: Delayed gadolinium-enhanced MRI of cartilage (dGEMRIC) in early knee osteoarthritis. Magn Reson Med 2003, 49(3):488-492.

16. Watanabe A, Wada Y, Obata T, Ueda T, Tamura M, Ikehira H, Moriya H: Delayed gadolinium-enhanced MR to determine glycosaminoglycan concentration in reparative cartilage after autologous chondrocyte implantation: preliminary results. Radiology 2006, 239(1):201-208.
17. Li W, Du H, Scheidegger R, Wu Y, Prasad PV: Value of precontrast T(1) for dGEMRIC of native articular cartilage. J Magn Reson Imaging 2009, 29(2):494-497

18. Williams A, Mikulis B, Krishnan N, Gray M, McKenzie C, Burstein D: Suitability of T(1Gd) as the dGEMRIC index at 1.5T and 3.0T. Magn Reson Med 2007, 58(4):830-834

19. Bittersohl B, Hosalkar HS, Kim YJ, Werlen S, Siebenrock KA, Mamisch TC: Delayed gadolinium-enhanced magnetic resonance imaging (dGEMRIC) of hip joint cartilage in femoroacetabular impingement (FAl): Are pre- and postcontrast imaging both necessary? Magn Reson Med 2009, 62(6):1362-1367.

20. Bellamy N, Buchanan WW, Goldsmith CH, Campbell J, Stitt LW: Validation study of WOMAC: a health status instrument for measuring clinically important patient relevant outcomes to antirheumatic drug therapy in patients with osteoarthritis of the hip or knee. J Rheumatol 1988, 15(12):1833-1840.

21. Wlberg G: Studies on dysplastic acetabula and congenital subluxation of the hip joint. With special reference to the complication of osteoarthritis. Acta Chir Scand 1939, 58(suppl):5-135.

22. Croft P, Cooper C, Wickham C, Coggon D: Defining osteoarthritis of the hip for epidemiologic studies. Am J Epidemiol 1990, 132(3):514-522.

23. Tonnis D: Normal values of the hip joint for the evaluation of $\mathrm{X}$-rays in children and adults. Clin Orthop Relat Res 1976, 119:39-47.

24. Burstein D, Velyvis J, Scott KT, Stock KW, Kim YJ, Jaramillo D, Boutin RD, Gray ML: Protocol issues for delayed Gd(DTPA)(2-)-enhanced MRI (dGEMRIC) for clinical evaluation of articular cartilage. Magn Reson Med 2001, 45(1):36-41.

25. Bittersohl B, Hosalkar HS, Werlen S, Trattnig S, Siebenrock KA, Mamisch TC: dGEMRIC and subsequent T1 mapping of the hip at 1.5 Tesla: normative data on zonal and radial distribution in asymptomatic volunteers. J Magn Reson Imaging 2011, 34(1):101-106.

26. Fujii M, Nakashima Y, Jingushi S, Yamamoto T, Noguchi Y, Suenaga E, Iwamoto $Y$ : Intraarticular findings in symptomatic developmental dysplasia of the hip. J Pediatr Orthop 2009, 29(1):9-13.

27. Jacobsen $S$, Romer L, Soballe K: Degeneration in dysplastic hips. A computer tomography study. Skeletal Radiol 2005, 34(12):778-784.

28. Shimogaki $K$, Yasunaga $Y$, Ochi M: A histological study of articular cartilage after rotational acetabular osteotomy for hip dysplasia. J Bone Joint Surg Br 2005, 87(7):1019-1023.

29. Yoshida $\mathrm{K}$, Azuma $\mathrm{H}$ : Contents and compositions of glycosaminoglycans in different sites of the human hip joint cartilage. Ann Rheum Dis 1982, 41(5):512-519.

30. Venn MF: Variation of chemical composition with age in human femoral head cartilage. Ann Rheum Dis 1978, 37(2):168-174.

31. Bashir A, Gray ML, Boutin RD, Burstein D: Glycosaminoglycan in articular cartilage: in vivo assessment with delayed Gd(DTPA)(2-)-enhanced MR imaging. Radiology 1997, 205(2):551-558.

doi:10.1186/1471-2474-13-212

Cite this article as: Xu et al.: Evaluation of radial distribution of cartilage degeneration and necessity of pre-contrast measurements using radial dGEMRIC in adults with acetabular dysplasia. BMC Musculoskeletal Disorders 2012 13:212

\section{Submit your next manuscript to BioMed Central and take full advantage of:}

- Convenient online submission

- Thorough peer review

- No space constraints or color figure charges

- Immediate publication on acceptance

- Inclusion in PubMed, CAS, Scopus and Google Scholar

- Research which is freely available for redistribution 